\title{
A Biography of Russell A. Johnson
}

\author{
George R. Sell · Yingfei Yi
}

Published online: 24 August 2011

(C) Springer Science+Business Media, LLC 2011

Russell A. Johnson was born in Fairmont, Minnesota on January 27, 1947. He obtained his B.S. degree in Mathematics from the University of Minnesota, Magna cum Laude, in 1969, and his Ph.D. degree in Mathematics from the University of Minnesota in 1975. His early academic career consisted of assistant professorships: one year at the University of Wisconsin-Parkside and 5 years in the Department of Mathematics, University of Southern California (USC). In 1981 he was promoted at USC, first to the rank of associate professor and then to a full professorship in 1988. At USC he served as the Vice-Director of Applied Mathematics (1986-1988) and as the Director of Graduate Programs in Mathematics (1988-1989). In 1991 he accepted a position of full professor at Universitá di Firenze in the Dipartimento di Sistemi e Informatica, which is his current academic home. While at Firenze, he also served as the Director of the Department (1995-1997). He has had visiting positions at the University of Minnesota (1980 and 1989-1990) and the Universität Heidelberg (1983-1985).

He has delivered numerous invited lectures at professional conferences and at universities/institutes worldwide. For example, he was a main lecturer at the C.I.M.E. School held in Cetraro, Italy in 2000, the DANCE Winter School held in Granada, Spain in 2007, and he has presented several mini-courses at Peking University (1990), University of Valladolid (2005), and East China Normal University (2010). In 2007 he was honored on his 60th birthday when the University of Valladolid in Spain hosted there an International Conference on Dynamical Methods and Mathematical Modelling.

Russell has made extensive services to the international mathematical community. Besides being on numerous professional committees, he is (or has been) a member of the editorial boards of the Journal of Dynamics and Differential Equations, Topological Methods in Nonlinear Analysis, Annali di Matematica Pura ed Applicata and six other professional journals. He has organized more than twenty international conferences, workshops, and

G. R. Sell

School of Mathematics, University of Minnesota, Minneapolis, MN 55455, USA

e-mail: sell@math.umn.edu

Y. Yi $(\varangle)$

School of Mathematics, Georgia Institute of Technology, Atlanta, GA 30332, USA

e-mail: yi@math.gatech.edu 
graduate summer schools including "Southern California Differential Equations Meeting" (Los Angeles 1978), "Differentialgleichungen und Dynamische Systeme" (Heidelberg 1983), "Differential and Dynamical Systems" (Los Angeles 1988), "Integrable Evolution Systems" (Los Angeles 1989), “C.I.M.E. Schools” (Montecatini Terme 1994 and Cetraro 2011), "Metodi Topologici ed Equazioni Differenziali" (Firenze 1996), "Comportamento Asintotico delle Soluzioni delle Equazioni Differenziali" (Bressanone 1999), "Qualitative Behavior of Differential Equations" (Certosa di Pontignano 2000), "Dynamical Methods for Differential Equations" (Medina del Campo 2002), "Dynamics of Cocycles and One-Dimensional Spectral Theory" (Oberwolfach 2005), and "Topological Methods, Differential Equations, Dynamical Systems" (Firenze 2007).

Russell is widely regarded as a world leader and pioneer in the dynamics of nonautonomous differential equations and skew-product flows. He has made widespread contributions, and his papers on topological dynamics and ergodic theory to differential equations are especially noteworthy. His mathematical works include contributions to the spectral theory of linear skew-product flows, recurrent Floquet theory, almost automorphic dynamics, spectral properties of almost periodic Schrödinger operators, and nonautonomous control theory. These areas are now widely studied and are among the most active areas of modern dynamics. Below is a brief summary of some of his important works in these and related areas.

Russell's early works after his Ph.D. concern topological dynamics and its measuretheoretic analogues. In [19], he constructed an example of minimal distal flow on the 3-torus which admits uncountably many invariant measures with nonisomorphic structure groups. He proved a disintegration result for invariant ergodic measures in a bitransformation group in [18], and in [17], he gave a generalization to the Peter-Weyl theory for the representation of a compact group acting on a locally convex topological vector space. In [21], he showed the existence of strong linear lifting of a compact flow when the acting group is either Abelian or a Lie group.

In the late 1970s, Russell began to work on the application of topological dynamics and ergodic theory to linear skew-product flows, especially to those arising in linear nonautonomous ordinary differential equations. With the introduction of the skew-product framework and the dynamical spectrum (now commonly referred to as the Sacker-Sell spectrum), this period marked an important development of the theory of nonautonomous dynamical systems, and Russell was among the major pioneers of this theory.

In [20], he gave a classification of the Sacker-Sell spectrum for a two-dimensional, linear differential system over a compact ergodic flow, where the spectrum is shown to be a single point in the elliptic case, two points in the hyperbolic case, and a nondegenerate interval otherwise. In the same work, the complementary ergodic bundles associated with a nondegenerate spectral interval is shown to be only measurable. In [22], Russell showed the smoothness of stable and unstable fibers associated with a resolvant point of the SackerSell spectrum for a nonlinear and nonautonomous system near the zero section. He adopted an operator-theoretic approach in [24] by considering a one-parameter group of differential operators generated by a nonautonomous linear system. He showed that the Sacker-Sell spectrum coincides with the real parts of the spectrum for the group, and, the spectral subbundles are analytic when the system depends analytically on the parameters. In the case that all solutions of a linear system are bounded and the coefficient space is distal and strictly ergodic, he showed in [24] and also in a joint work with Ellis [4] that the system is kinematically similar to a system with skew-Hermitian coefficients. In a joint work [51] of Russell with Palmer and Sell, deep connection among the Sacker-Sell spectrum, the multiplicative ergodic theorem, and Lyapunov exponents was established for a linear nonautonomous system. 
In [35], Russell discovered a generic property of exponential dichotomy for trace-free, quasiperiodic linear systems parametrized by both continuously varying coefficients and varying frequencies. Later Russell with Fabbri [6] showed that this property is in fact both open and dense. Such a generic property was shown by Russell with Yi [48] to play an important role in studying generic, intermittent Hopf bifurcations from invariant tori when a family of spectral intervals cross zero as the parameters vary. This study was later extended by Russell [38] and also by Russell with Kloeden and Pavani [61] to random, two-step bifurcations. In two recent works [5,13] of Russell with Fabbri and Zampogni, the generic property of exponential dichotomy for trace-free, quasiperiodic linear systems is shown to be closely related to the density of positive Lyapunov exponents.

The existence of nondegenerate intervals in the Sacker-Sell spectrum for a linear almost periodic system suggests that there is a significant difference from a linear periodic system. While noting this, Russell went further to investigate the oscillatory behavior of solutions by measuring their rotational effects in order to capture the information lacking in the SackerSell spectral theory. In two-dimensions and in connection with the study on Floquet theory in almost periodic cases, he introduced a useful technique by relating such oscillatory behavior to the dynamics of the projective flow induced by the system. Using this technique, he made a systematic investigation of the mechanism that leads to the failure of a full almost periodic Floquet theory in two-dimensional almost periodic linear systems, by constructing various examples concerning the existence of an almost automorphic (but not almost periodic) set in the projective flow. These examples include various cases with respect to either degenerate or nondegenerate Sacker-Sell spectra, unique or nonunique minimal sets in the associated projective flows, and either resonant or nonresonant rotation numbers $[26,30,33]$.

In [23], he showed that the projective flow associated with a linear quasiperiodic system in two-dimension with rotational coefficients matrices can be foliated into measurable, noncontinuous, invariant one-dimensional subbundles. In [28], he constructed an example of almost periodic linear system in two-dimension in which the associated projective flow is a minimal proximal extension of the base flow. With this example, as later discovered by Russell with Bjerklov [1] and Huang and Yi, one has the only known example of a continuous flow with Li-Yorke chaos.

In 1980, Russell wrote an important paper [25] on Floquet theory of an almost periodic linear system in two-dimension by relating Floquet property to the almost automorphic minimal dynamics of the projective flow induced by the system. With the advantage of hindsight, one can now see that the resulting insights developed by Russell and others and using the knowledge of [25], which in itself is concerned with a very simple model (2D and linear), has played a profound role in many of the subsequent developments in the study of the dynamics and applications of general linear nonautonomous systems. A remarkable finding in this paper is the general unavailability of an almost periodic strong Perron transformation which transforms an almost periodic linear differential system into a canonical form. However, there do exist almost automorphic transformations instead. The paper [25] has proven to be a techtonic shift in the dynamical landscape.

With Sell, he also studied in [47] some valid cases of quasiperiodic Floquet theory in quasiperiodic linear systems with Diophantine frequencies when the spectral subbundles are sufficiently smooth and a full Sacker-Sell spectrum condition is satisfied. This is in fact the first work in the literature towards the study of quasiperiodic Floquet theory or reducibility.

Almost automorphy is a notion generalizing almost periodic functions introduced by S. Bochner in 1955 in a differential geometry context which had been mainly studied in a pure topological dynamics context. Besides his work in almost automorphic Floquet theory, Russell in several other original works in the early 1980s demonstrated the usefulness 
of this notion in characterizing oscillatory dynamics of almost periodic differential equations. Since the 1950s, accompanied by the development of topological dynamics and theory of skew-product flows, there has been a vast number of papers on finding almost periodic solutions and studying their stability, for differential equations with almost periodic time dependence. It was noticed that almost periodic systems differ significantly from either the autonomous or the periodic systems because of the lack of existence of almost periodic solutions in many almost periodic systems. This results in a question: What is the fundamental nature of oscillatory solutions of those almost periodic systems that have no almost periodic solutions?

To answer this question, one needs to examine the nature of recurrent solutions that respond to the harmonics of the almost periodic time dependence. In [29], Russell constructed an example of scalar, almost periodic, linear nonhomogeneous equation in which there is a unique bounded solution and that this solution is almost automorphic but not almost periodic. Later in [27], he showed that the coefficients of such equations not only form a residual set in the hull of the coefficient space, but also they have full Haar measure. Stimulated by these findings and other related works of Russell, later studies by Shen and Yi and many others have shown that almost automorphic phenomenon is a fundamental property occurring in almost periodically forced differential equations. In [40], Russell and Mantellini also showed that almost automorphic dynamics plays an important role in transcritical bifurcation problems of quasiperiodic systems.

The spectral theory of Schrödinger operators with almost periodic (or recurrent) potentials is another important area in which Russell was a pioneer with major contributions. In contrast to the case of a periodic potential, in which the spectrum is known to be a union of nondegenerate intervals, it had been conjectured that Schrödinger operators with almost periodic potentials should typically admit singular continuous spectra with Cantor structures. By using dynamical characteristics such as Sacker-Sell spectrum, Lyapunov exponents and rotation numbers, Russell introduced a novel dynamical systems approach to the study of these spectral problems.

In 1982, Russell wrote a beautiful and seminal paper [41] with Moser on almost periodic Schrödinger operators in which the rotation number is defined and shown to be continuous, and monotonically increasing precisely on the spectrum. This result, now widely recognized as the Gap Labeling Theorem, plays a central role in the study of spectral problems for Schrödinger operators, especially when studying problems with nonsmooth recurrent potentials. The work [41] also introduces a useful notion of Floquet exponent by combining the Lyapunov exponents with complex rotation numbers. In [32], Russell further explored the property of Lyapunov exponents for an almost periodic Schrödinger operator by showing its harmonicity in the resolvent of the spectrum and its one-sided continuity at an endpoint of a spectral gap.

With Giachetti in [16], he extended the notions and properties of rotation numbers and Floquet exponents to general Schrödinger-like operators by making use of Titchmarsh-Weyl $m$-functions. In [34], he introduced a higher dimensional geometric theory of rotation numbers and Floquet exponents and also proved a spectral gap labeling theorem. In [37], he generalized the notion of rotation numbers to Schrödinger operators in higher spatial dimension to obtain a Maslov-like index. With Nerurkar in [44], he extended the concept of rotation number in higher dimension defined in [37] to linear nonautonomous Hamiltonian systems. With Fabbri and Núnẽz in [8,9] and Novo and Obaya in [59,60], he developed a general spectral theory in particular gap-labelling theory for nonautonomous linear Hamiltonian systems by introducing a notion of Floquet exponents and studying properties of Lyapunov exponents, exponential dichotomy, and Weyl $m$-functions. 
In [31], he made a deep connection between the spectrum of a Schrödinger operator with an ergodic, recurrent potential and the Sacker-Sell spectrum of the corresponding linear system and showed that the Schrödinger spectrum admits no isolated point. Positivity of Lyapunov exponents over a spectral interval was also shown and an inverse problem describing the algebraic-geometric Sturm-Liouville coefficients was treated when the spectrum is a finite union of nondegenerate intervals. Such inverse problems were generalized to random ergodic AKNS operators by Russell with De Concini in [3] and to higher dimensions in a recent work [49] of Russell with Zampogni in which it is shown that the ergodic coefficients triples can be described using a generalized Jacobian variety when the spectrum is a finite union of nondegenerate intervals with vanishing Lyapunov exponent. In a succeeding work [50] of Russell with Zampogni, the inverse spectral problem is studied for the Camassa-Holm equation where the spectral problem is the so-called acoustic equation.

In [36], Russell proved a generic property of an exponential dichotomy for linear systems associated with quasiperiodic Schrödinger operators. As a consequence, he showed the genericity of Cantor spectrum of quasiperiodic Schrödinger operators when both potential functions and their frequencies are varying.

In the early 1990s, Russell began to work on control problems involving time-varying coefficients. With Nerurkar in [43], Russell showed that for a linear nonautonomous control system local null controllability implies global null controllability under some conditions on the Sacker-Sell spectrum. In [45], they used the spectral theory of linear nonautonomous Hamiltonian systems and the theory of exponential dichotomies to characterize feedback stabilization for linear nonautonomous control processes. Some improvements of these results were made by Russell with Colonius in [2]. A more systematic study of the nonautonomous control problems was made by Russell and Nerurkar in [46] in which it is shown that a nonautonomous system having positive Lyapunov exponents can still be globally null-controllable. The theory of nonautonomous control, which Russell developed with collaborators, was later applied by Russell with Fabbri and Kloeden in [7] to digitization or discretization problems for linear, nonautonomous control. In several recent works of Russell with Fabbri, Impram, Novo, and Núnẽz [14,10,11], the general spectral theory of linear nonautonomous Hamiltonian systems which Russell developed with collaborators is applied to nonautonomous control problems, for instance to the generalization of the Yakubovich frequency theorem to nonautonomous control systems.

Russell has other broad mathematical interests. For example, he has worked on the existence and asymptotics of grand states for semilinear elliptic equations in unbounded domains [15,52-55], periodic solutions of damped wave equations and Navier-Stokes equations in thin domains [56-58], Sharkovskii ordering for almost periodically forced interval maps [12], and the nature of nonautonomous global attractors [39,42].

In closing, we make some personal comments about our experiences in working with Russell.

(GRS): Our mathematical saga began when Russell was writing his Ph.D. thesis at the University of Minnesota. He was attending my lectures on nonautonomous dynamics, and he became very interested in my evolving theory on dynamics with Robert Sacker (at USC). Russell and I formed a small seminar-of-two, where Russell became an unofficial "predoctoral fellow." Our collaborations continued after Russell received his Ph.D. in 1975.

That year, like today, was a difficult time for finding an academic job. Fortunately, Russell found a teaching position at a small college in southern Wisconsin. This location was not far from the home of my parents. During my many visits to southern Wisconsin, Russell and I continued our seminar, while frequenting various coffee shops in the Milwaukee area! 
During our collaborations, we became interested in studying the mathematical connections between the spectral theory based on the Sacker-Sell spectrum and Lyapunov spectrum, which arises in the Multiplicative Ergodic Theorem (MET). The results of this study, written in our joint work with Palmer [51], include: (1) a new proof of the MET that is based on the Perron Triangularization Theorem; (2) that the measurable foliation in the MET is a refinement of the continuous foliation given by the Sacker-Sell theory; (3) that the endpoints of the Sacker-Sell spectral intervals are in the Lyapunov spectrum; and (4) that one can calculate the Lyapunov spectrum, including the multiplicities of repeated growth rates, without the need of an eigen-basis of Lyapunov vectors. It is noteworthy that the paper [51], which was published over 30 years ago, is of current use in many places today, including, for example, a recent preprint by GRS (and others) on "Ensemble dynamics and bred vectors."

(YY): The year 1987 marked the beginning of my career as a researcher in mathematics when I became a Ph.D. student of Russell at USC. At the time, due to an insufficient number of students enrolled, there was neither a graduate ODE course nor a dynamical systems course offered at USC. This caused great difficulty for a foreign student with a non-mathematical background like me who wished to study dynamics. In Fall 1987, Russell organized a student ODE seminar which, in particular, included a minicourse on classical ODE theory taught by Russell himself. It was through this seminar that I was attracted to the world of dynamics and differential equations.

I began to work on my Ph.D. thesis in 1988 under the guidance of Russell. At the time, Russell was interested in nonlinear dynamical behaviors of differential equations especially those involving either nonautonomous forcing or internal multifrequencies. Motivated by his discovery of generic property of exponential dichotomy for trace-free, quasiperiodic linear systems, he conjectured that higher dimensional Hopf bifurcation in a generic sense should behave very differently than that in the traditional sense. The investigation of this problem later became a part of my Ph.D. thesis topics. It was through working on this thesis that I learned so much in dynamical systems theory, ranging from invariant manifolds, perturbations and bifurcations, to nonautonomous dynamics, as well as to small divisor problem.

In 1989, Russell was invited, as a visiting professor of the Institute for Mathematics and its Applications (IMA) at the University of Minnesota, to participate in the 1989-1990 annual dynamical systems program held at the IMA. He supported me with a research assistantship and made arrangements with the IMA for me to also participate in the annual program. Participating in this program turned out to be the best experience I have ever had in my career. Not only was I exposed to diversified areas of modern dynamical systems, but also I benefited tremendously from participating in conferences and workshops contained in the program. In addition, participating in the program provided me with a unique opportunity to learn from and interact with top researchers working in various areas of dynamics.

I have had many fruitful collaborations with Russell, both before and after I obtained my $\mathrm{Ph} . \mathrm{D}$. degree in 1990. In our first joint work [48], we showed that Hopf bifurcation from higher dimensional tori enjoys an intermittency property in which bifurcation to equal dimensional tori occurs generically. Later, Russell and I collaborated extensively with Xingbin Pan on the existence and asymptotics of grand states of semilinear elliptic equations in unbounded domains [52-55]. In these works, we introduced some dynamical systems techniques into the study of radially symmetric positive solutions of semilinear elliptic equations in unbounded domains.

I am indebted to Russell for his constant support and inspiration over the years to my career development. 


\section{References}

1. Bjerklov, K., Johnson, R.A.: Minimal subsets of projective flows. Discrete Continuous Dyn. Syst. Ser. B 9, 495-516 (2008)

2. Colonius, F., Johnson, R.A.: Local and global null controllability of time varying linear control systems. ESAIM Control Optim. Calc. Var. 2, 329-341 (1997)

3. De Concini, C., Johnson, R.A.: The algebraic-geometric AKNS potentials. Ergod. Theory Dyn. Syst. 7, 1-24 (1987)

4. Ellis, R., Johnson, R.A.: Topological dynamics and linear differential systems. J. Differ. Equ. 44, 2139 (1982)

5. Fabbri, R., Johnson, R.A.: On the Lyapunov exponent of certain $s l(2, \mathbb{R})$-valued cocycles. Differ. Equ. Dyn. Syst. 7, 349-370 (1999)

6. Fabbri, R., Johnson, R.A.: Genericity of exponential dichotomy for two-dimensional differential systems. Ann. Mat. Pura Appl. 178, 175-193 (2000)

7. Fabbri, R., Johnson, R.A., Kloeden, P.E.: Digitization of nonautonomous control systems. J. Differ. Equ. 195, 210-229 (2003)

8. Fabbri, R., Johnson, R.A., Núnẽz, C.: Rotation number for nonautonomous linear Hamiltonian systems I. Basic properties. Z. Angew. Math. Phys. 54, 484-502 (2003)

9. Fabbri, R., Johnson, R.A., Núnẽz, C.: Rotation number for nonautonomous linear Hamiltonian systems II. The Floquet coefficients. Z. Angew. Math. Phys. 54, 652-676 (2003)

10. Fabbri, R., Johnson, R.A., Núnẽz, C.: On the Yakubovich frequency theorem for linear non-autonomous control processes. Discrete Continuous Dyn. Syst. 9, 677-704 (2003)

11. Fabbri, R., Impram, S.T., Johnson, R.A.: On a criterion of Yakubovich type for the absolute stability of nonautonomous control processes. Int. J. Math. Math. Sci. 16, 1027-1041 (2003)

12. Fabbri, R., Jäger, T., Johnson, R.A., Keller, G.: A Sharkovskii-type theorem for minimally forced interval maps. Topol. Methods Nonlinear Anal. 26, 163-188 (2005)

13. Fabbri, R., Johnson, R.A., Zampogni, L.: On the Lyapunov exponent of certain $S L(2, \mathbb{R})$-valued cocycles II. Differ. Equ. Dyn. Syst. 18, 135-161 (2010)

14. Fabbri, R., Johnson, R.A., Novo, S., Núnẽz, C.: Some remarks concerning weakly disconjugate linear Hamiltonian systems. J. Math. Anal. Appl. 380, 853-864 (2011)

15. Franca, M., Johnson, R.A.: Ground states and singular ground states for quasilinear partial differential equations with critical exponent in the perturbative case. Adv. Nonlinear Stud. 4, 93-120 (2004)

16. Giachetti, R., Johnson, R.A.: The Floquet exponent for two-dimensional linear systems with bounded coefficients. J. Math. Pures Appl. 65, 93-117 (1986)

17. Johnson, R.A.: Representations of compact groups on topological vector spaces: some remarks. Proc. Am. Math. Soc. 61, 131-136 (1976)

18. Johnson, R.A.: Disintegration of measures on compact transformation groups. Trans. Am. Math. Soc. 233, 249-264 (1977)

19. Johnson, R.A.: The measure-theoretic structure group is not invariant. Illinois J. Math. 21, 178-184 (1977)

20. Johnson, R.A.: Ergodic theory and linear differential equations. J. Differ. Equ. 28, $23-34$ (1978)

21. Johnson, R.A.: Existence of a strong lifting commuting with a compact group of transformations. Pac. J. Math. 76, 69-81 (1978)

22. Johnson, R.A.: Concerning a theorem of Sell. J. Differ. Equ. 30, 324-339 (1978)

23. Johnson, R.A.: Measurable subbundles in linear skew-product flows. Illinois J. Math. 23, 183-198 (1979)

24. Johnson, R.A.: Analyticity of spectral subbundles. J. Differ. Equ. 35, 366-387 (1980)

25. Johnson, R.A.: On a Floquet theory for almost periodic, two dimensional linear systems. J. Differ. Equ. 37, 184-205 (1980)

26. Johnson, R.A.: Linear differential equations with interval spectrum. Proc. Am. Math. Sci. 80, 90-94 (1980)

27. Johnson, R.A.: Bounded solutions of scalar, almost periodic linear equations. Illinois J. Math. 25, 632-643 (1981)

28. Johnson, R.A.: Two-dimensional, almost periodic linear systems with proximal and recurrent behavior. Proc. Am. Math. Soc. 82, 417-422 (1981)

29. Johnson, R.A.: A linear, almost periodic equation with an almost automorphic solution. Proc. Am. Math. Soc. 82, 199-205 (1981)

30. Johnson, R.A.: On almost-periodic linear differential systems of Millionščikov and Vinograd. J. Math. Anal. Appl. 85, 452-460 (1982)

31. Johnson, R.A.: The recurrent Hill's equation. J. Differ. Equ. 46, 165-193 (1982)

32. Johnson, R.A.: Lyapounov numbers for the almost periodic Schrödinger equation. Illinois J. Math. 28, 397-419 (1984) 
33. Johnson, R.A.: An Example Concerning the Geometric Significance of the Rotation Number-Integrated Density of States, Lyapunov Exponents (Bremen, 1984). Lecture Notes in Mathematics, vol. 1186, pp. 216-226. Springer, Berlin (1986)

34. Johnson, R.A.: $m$-functions and Floquet exponents for linear differential systems. Ann. Mat. Pura Appl. 147, 211-248 (1987)

35. Johnson, R.A.: Hopf bifurcation from nonperiodic solutions of differential equations: I. Linear theory. J. Dyn. Differ. Equ. 1, 179-198 (1989)

36. Johnson, R.A.: Cantor spectrum for the quasi-periodic Schrödinger equation. J. Differ. Equ. 91, 88-110 (1991)

37. Johnson, R.A.: Oscillation theory and the density of states for the Schrödinger operator in odd dimension. J. Differ. Equ. 92, 145-162 (1991)

38. Johnson, R.A.: An application of topological dynamics to bifurcation theory. In: Topological dynamics and applications (Minneapolis, MN, 1995). Contemporary Mathematics, 215, pp. 323-334. American Mathematical Society, Providence (1998)

39. Johnson, R.A., Kloeden, P.E.: Nonautonomous attractors of skew-product flows with digitized driving systems. Electron J Differ. Equ. 58, 16 (2001)

40. Johnson, R.A., Mantellini, F.: A nonautonomous transcritical bifurcation problem with an application to quasi-periodic bubbles. Discrete Continuous Dyn. Syst. 9, 209-224 (2003)

41. Johnson, R.A., Moser, J.: The rotation number for almost periodic potentials. Commun. Math. Phys. 84, 403-438 (1982)

42. Johnson, R.A., Munoz-Villarragut, V.: Some questions concerning attractors for nonautonomous dynamical systems. Nonlinear Anal. TMA 71, 1858-1868 (2009)

43. Johnson, R.A., Nerurkar, M.: On null controllability of linear systems with recurrent coefficients and constrained control. J. Dyn. Differ. Equ. 4, 259-273 (1992)

44. Johnson, R.A., Nerurkar, M.: Exponential dichotomy and rotation number for linear Hamiltonian systems. J. Differ. Equ. 108, 01-216 (1994)

45. Johnson, R.A., Nerurkar, M.: Stabilization and linear regulator problem for random linear control process. J. Math. Anal. Appl. 197, 608-629 (1996)

46. Johnson, R.A., Nerurkar, M.: Controllability, stabilization and the regulator problem for random differential systems. Memoirs of the American Mathematical Society 136, no. 646 (1998)

47. Johnson, R.A., Sell, G.R.: Smoothness of spectral subbundles and reducibility of quasi-periodic linear differential systems. J. Differ. Equ. 41, 262-288 (1981)

48. Johnson, R.A., Yi, Y.: Hopf bifurcation from nonperiodic solutions of differential equations: II. J. Differ. Equ. 107, 310-340 (1994)

49. Johnson, R.A., Zampogni, L.: Description of the algebro-geometric Sturm-Liouville coefficients. J. Differ. Equ. 244, 716-740 (2008)

50. Johnson, R.A., Zampogni, L.: On the Camassa-Holm and K-dV hierarchies. J. Dyn. Differ. Equ. 22, 331-366 (2010)

51. Johnson, R.A., Palmer, K.J., Sell, G.R.: Ergodic properties of linear dynamical systems. SIAM J. Math. Anal. 18, 1-33 (1987)

52. Johnson, R.A., Pan, X., Yi, Y.: Singular ground states of semi-linear elliptic equations via invariant manifolds theory. Nonlinear Anal. TMA 20, 1279-1302 (1993)

53. Johnson, R.A., Pan, X., Yi, Y.: Positive solutions of super-critical elliptic equations and asymptotics. Commun. Partial Differ. Equ. 18, 977-1019 (1993)

54. Johnson, R.A., Pan, X., Yi, Y.: The Melnikov method and elliptic equations with critical exponent. Indiana Univ. Math. J. 43, 1045-1077 (1994)

55. Johnson, R.A., Pan, X., Yi, Y.: Singular solutions of elliptic equation $\Delta u-u+u^{p}=0$. Annali di Matematicá prera ed Applićata (IV) CLXVI, 203-225 (1994)

56. Johnson, R.A., Nistri, P., Kamenski, M.: On periodic solutions of a damped wave equation in a thin domain using degree-theoretic methods. J. Differ. Equ. 140, 186-208 (1997)

57. Johnson, R.A., Nistri, P., Kamenski, M.: Bifurcation of periodic solutions of the Navier-Stokes equations in a thin domain. Topol. Methods Nonlinear Anal. 13, 281-300 (1999)

58. Johnson, R.A., Nistri, P., Kamenski, M.: On the existence of periodic solutions of the Navier-Stokes equations in a thin domain using the topological degree. J. Dyn. Differ. Equ. 12, 681-712 (2000)

59. Johnson, R.A., Novo, S., Obaya, R.: Ergodic properties and Weyl $M$ M-functions for random linear Hamiltonian systems. Proc. R. Soc. Edinb Sect. A 130, 1045-1079 (2000)

60. Johnson, R.A., Novo, S., Obaya, R.: An ergodic and topological approach to disconjugate linear Hamiltonian systems. Illinois J. Math. 45, 803-822 (2001)

61. Johnson, R.A., Kloeden, P.E., Pavani, R.: Two-step transition in nonautonomous bifurcations: an explanation. Stoch. Dyn. 2, 67-92 (2002) 